\title{
Synchronizing Renewable Energy Sources in Distributed Generation Systems
}

\author{
C. Ramos $^{1}$, A. Martins ${ }^{1}$ and A. Carvalho ${ }^{1}$ \\ ${ }^{1}$ Department of Electrical Engineering and Computers \\ Faculty of Engineering of the University of Porto \\ Rua Dr Roberto Frias, s/n, 4200-465, Porto, (Portugal) \\ Phone: +351225081507, Fax: +351225081443, email: cjr@fe.up.pt, ajm@fe.up.pt, asc@fe.up.pt
}

\begin{abstract}
In renewable and distributed generation systems the grid voltage quality can be poor. So, the connection of power converters to the grid requires accurate control of synchronism between the two sources. The paper discusses issues on synchronization of power converters connected to the mains and related control methods. The design and experimentation of a prototype of a $200 \mathrm{~kW}$ power converter that connects a wind generator to the grid help to validate the adopted approach. In this paper a new zero crossing synchroniser is proposed having zero error in steady state, moderate speed response to grid frequency changes and amplitude voltage variations, and harmonics and noise immunity. The speed response, and harmonics and noise immunity are a trade-off. Simulation results with strong disturbances in the grid voltage show the robustness of the proposed method.
\end{abstract}

\section{Keywords}

Renewable energy, power quality, distributed generation, weak grid, signal processing

\section{Introduction}

The interconnection of power converters to the grid requires accurate control of synchronism between converter and grid. This requirement becomes a critical one for systems in that voltage quality of grid is not good. This is the case for renewal generation if the location of the plant is either a remote one or an island. This has caused some research on developing synchronization methods that minimally are affected by this poor power quality.

The paper discusses issues on synchronization of power converters connected to Mains and related control methods. The design and experimentation of a prototype of a $200 \mathrm{~kW}$ power converter that connects a wind generator to the grid help to validate the adopted approach. The main goal is to compute in real time an accurate estimate of the phase-angle $(\theta)$ of the grid voltages.
The environment of design may be summarized as: wind generator is located in an island with a grid presenting bad characteristics - voltage sags and swells are frequent and heavy, the harmonic components are considerably high, and the frequency presents some oscillation around the $50 \mathrm{~Hz}$. Some grid synchronizers reported in literature are done with a Phase Locked Loop [2]-[3] having the inherent slower response particularly if they use zero cross detection, others are based on the d-q transformation theory [4] having instant updates but are more sensitive to grid disturbances if the filtering is low to achieve fast response. In this paper a new zero crossing synchronizer is proposed having zero error in steady state, moderate speed response to grid frequency changes and amplitude voltage variations, and harmonics and noise immunity. The speed response, and harmonics and noise immunity are a tradeoff.

\section{Disturbances on Phase Detection}

Synchronization is an operation based on detection of phase. Disturbances on voltage waveforms cause phase changes and difficulties on designing a simple method for synchronizing different waveforms. This synchronizer is based on the zero crossing detection of the grid voltages. The three phases provide six zero crossings, so the phase-angle and frequency are verified six times per period. If the lower order harmonics are not in phase, the zero crossings are changed, and if they have a high amplitude cause extra zero crossings detections. The lower order harmonics effects in zero crossings detection can be highly reduced through inclusion of a low pass digital filter.

The high frequency harmonics can also produce an error in zero crossings detection but this problem can be eliminated or highly reduced through appropriate anti-aliasing analog filters. The effect of the voltages spikes and notches are highly reduced using a digital filter as well as specific sampling techniques in which each voltage sample is an average of the voltage during 
samplings. This can be done by an analog integrator reseted after the analog to digital conversion or by an over sampling. This technique also reduces the noise in the measurements.

The following sections are centered at analyzing these issues in order to design an accurate and fast synchronizer capable of operating within a weak grid disturbed by different non-linear operating conditions.

\section{Zero Crossing Detection}

The zero cross detection is based on the measured line to line voltages $\left(V_{R S}, V_{S T}, V_{T R}\right)$ required by the control algorithm. If only two voltages are measured the other is computationally obtained. As stated in section 2 the harmonics, noise, voltages spikes and notches are highly reduced by the anti-aliasing analog filters, by using the average voltage between samples instead of the instant voltage value, and by a digital filter. Using a FIR (Finite Impulse Response) filter the time delay is constant, so the phase angle delay is linear and easily computed in order to compensate the delay. The filter order must be a compromise between the delay and the harmonics attenuation. The phase delays introduced by the filter $\left(\theta_{f}[\mathrm{rad}]\right)$ and by the use of the average samples $\left(\theta_{a}[\mathrm{rad}]\right)$ are given by eq. 1 , where $f_{s}[\mathrm{~Hz}]$ is the sampling frequency, $f[\mathrm{~Hz}]$ the grid frequency, and $N_{f}$ the FIR filter order [1].

$$
\begin{aligned}
& \theta_{f}=\pi\left(N_{f}-1\right) f / f_{s} \\
& \theta_{a}=\pi f / f_{s}
\end{aligned}
$$

At every sample instant $(j)$, whenever a change in sign of two consecutive filtered measures $\left(S_{j}\right)$ in the same voltage is detected, a zero cross of the respective voltage is obtained (fig. 1).

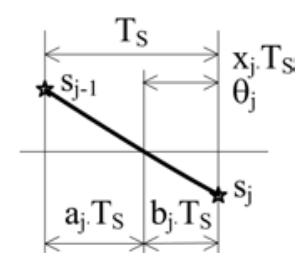

a)

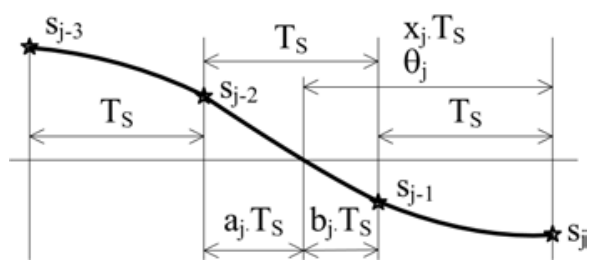

b)

Fig. 1. Zero crossing detection with: a) two samples, and b) four samples.

If the sampling frequency is high enough, the phaseangle in sampling period $j$ can be determined by assuming that the signal $S(t)$ is a straight line between the sampling points. In this case the values $a_{j}, b_{j}$ and $\theta_{j}$ (fig. 1a) are obtained through eq. 2 .

$$
\begin{aligned}
& a_{j}=\frac{S_{j-1}}{S_{j-1}-S_{j}} ; \\
& b_{j}=1-a_{j} ; \\
& \omega t_{j}=2 \pi b_{j} \frac{T_{s}}{T}+\theta_{C P}
\end{aligned}
$$

where $\theta_{C P}$ is a value that depends on the phase where the zero crossing was detected and is given by Table 1 .

Table 1: Offset angle, with origin in phase R.

\begin{tabular}{|c|c|}
\hline Zero cross detected Phase & $\theta_{C P}[\mathrm{rad}]$ \\
\hline Descending Phase TR & $\pi / 6$ \\
\hline Ascending Phase ST & $3 \pi / 6$ \\
\hline Descending Phase RS & $5 \pi / 6$ \\
\hline Ascending Phase TR & $7 \pi / 6$ \\
\hline Descending Phase ST & $9 \pi / 6$ \\
\hline Ascending Phase RS & $11 \pi / 6$ \\
\hline
\end{tabular}

If the sampling frequency is not high enough, a better approach is to assume that the signal $S(t)$ is a polynomial defined by four consecutive samples (two less than zero and two greater than zero) (fig. 1b).

Using this polynomial, to obtain the value $a_{j}$ through an explicit formula, a large number of computations would be required. To reduce the computations, an iterative solution is find. A good solution is found with only one iteration if the initial value is taken from the middle samples. In this case the equations to find $\theta_{j}$ are represented in eq. 3 .

$$
\begin{aligned}
& a_{0}=\frac{S_{j-2}}{S_{j-2}-S_{j-1}} \\
& a_{j}=\frac{k_{1} a_{0}^{3}+k_{2} a_{0}^{2}+6 S_{j-2}}{3 S_{j-2}-6 S_{j-1}+2 S_{j-3}+S_{j-0}} \\
& b_{j}=1-a_{j} ; \omega t_{j}=2 \pi\left(b_{j}+1\right) \frac{T_{s}}{T}+\theta_{C P}
\end{aligned}
$$

where $\quad k_{1}=3 S_{j-2}-3 S_{j-1}+S_{j-0}-S_{j-3} \quad$ and $k_{2}=3 S_{j-1}+3 S_{j-3}-6 S_{j-2}$.

Even after some manipulation to reduce the computation, eq. 3 is time consuming compared with eq. 2. Figure 2 shows the maximum error on $\theta$ (in degrees) determined by eq. 2 and eq. 3 in function of the relation between sampling frequency and grid frequency.

A more complex filtering can be done using predictive filtering as described in [5]. 


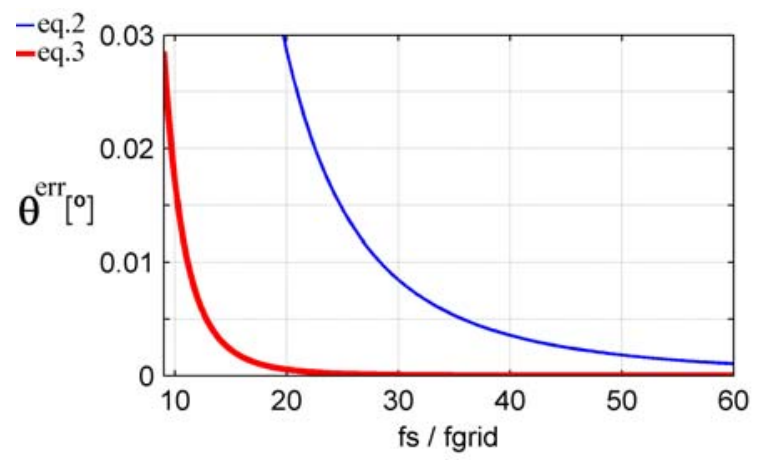

Fig. 2. Maximum error in $\theta$ (in degrees) determined by eq. 2 and eq. 3 .

\section{Frequency and Phase Angle Estimation}

The grid period is obtained by taking the time between two consecutive zero crossings. This is done with a counter to obtain the number of $j$ samples between two consecutives zero crossings and by the values $a_{i}$ and $b_{i \text { - }}$ ${ }_{1}$, where $i$ indicates the zero crossing detection order as is shown in fig. 3 .

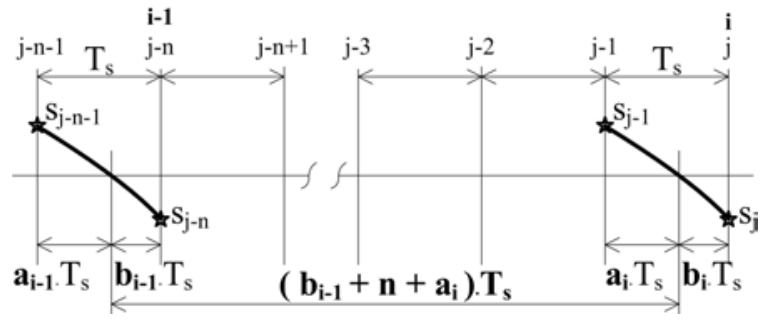

Fig. 3. Grid period calculation from two consecutive zero crossings.

This way, the grid period $T_{i}$ is taken from eq. 4 six times per period (in the three phase systems case) where $a_{i}$ and $b_{i-1}$ are obtained as explained in section 3, $n_{i}$ the number of $j$ samples counted, and $T_{a}$ the sampling period. The grid frequency $\left(f_{i}\right)$ is the inverse of $T_{i}$.

$$
\begin{aligned}
T_{i} & =6\left(b_{i-1}+n_{i}+a_{i}\right) T_{a} ; \\
f_{i} & =1 / T_{i}
\end{aligned}
$$

The phase angle estimation in the sample instant $j\left(\hat{\theta}_{j}\right)$ is obtained by increment every sample instant its value by the value $\Delta_{i}$ as indicated in eq. 5 . Thus, $\Delta_{i}$ needs to be computed in order to maintain the phase angle error ( $\theta_{j}^{\text {err }}$ ) null every instant $j . \theta_{j}$ is only known in the zero crossings instants $i$ and its value is given by eq. 2 or eq. 3 as indicated in section 3 . Note that $i$ represents the instants when a zero crossing is detected.

$$
\begin{aligned}
& \theta_{j}^{e}=\theta_{j-1}^{e}+\Delta_{i} ; \\
& \theta_{j}^{e r r}=\theta_{j}-\hat{\theta}_{j}
\end{aligned}
$$

Assuming that the measured grid period in $i\left(T_{i}\right)$ is maintained during the time interval between $i$ and $i+1$, $T_{i+1}^{*}=T_{i}$ the number of instant samples $j$ between $i$ and $i+1$ will be the same: $n_{i+1}^{*}=n_{i}$. Thus the expected value of $\theta_{i+1}$ in the next zero crossing is given by eq. 6 (the superscript $*$ denotes assumption values).

$$
\theta_{i+1}^{*}=\theta_{i}+n_{i} 2 \pi \frac{T_{s}}{T_{i}}
$$

With the same assumption, the phase angle estimation in the next zero crossing instant is given by eq. 7 .

$$
\theta_{i+1}^{e^{*}}=\theta_{i}^{e}+n_{i} \Delta_{i+1}
$$

Now the goal is to make $\theta_{i+1}^{* e}=\theta_{i+1}^{*}$, that is, in the next zero crossing the phase angle estimation is expected to be the same as the grid phase angle. Due to the phase angle circular nature $(\theta \in[-\pi \ldots \pi]), \Delta_{i}$ is calculated to speed up or slow down the phase angle in order to $\hat{\theta}$ converge to $\theta$ by the shortest path as shown in eq. 8 .

$$
\Delta_{i+1}=\left\{\begin{array}{lll}
\left(\theta_{i+1}^{*}-\hat{\theta}_{i}\right) / n_{i} & \text { if } & \hat{\theta}_{i}<\theta_{i+1}^{*}+\pi \\
\left(\theta_{i+1}^{*}+\pi-\hat{\theta}_{i}\right) / n_{i} & \text { if } & \hat{\theta}_{i} \geq \theta_{i+1}^{*}+\pi
\end{array}\right.
$$

\section{Performance of Synchronism Method}

This approach for a synchronizer operating in a hard environment is validated through analysis of results carried out within Saber Designer tool. Figures 4 to 8 show the results of the proposed synchronizer operating in different conditions of grid voltage. The sampling frequency is $1 \mathrm{kHz}$, each sample is obtained by averaging 32 over-samplings and the filter is a $9^{\text {th }}$ order Low Pass Equiripple FIR, except in fig. 4 where no filter is applied.

The grid voltage $\left(V_{R S}, V_{S T}, V_{T R}\right)$ represents the line to line voltage, $f$ the grid frequency, $\hat{f}$ the estimated grid frequency, $\theta$ the grid phase-angle, $\hat{\theta}$ the estimated grid phase-angle and $\theta-\hat{\theta}$ the estimated grid phase angle error magnified 10 times. Figure 4 shows that in a clear grid (without harmonics, spikes and notches) the filter is not required and the synchronizer is not affected by a voltage sag. In the other simulations a filter is used to allow the synchronizer to work and the results are presented in Fig. 5 to 9. Figure 5 presents the filter influence on the phase angle estimation. 


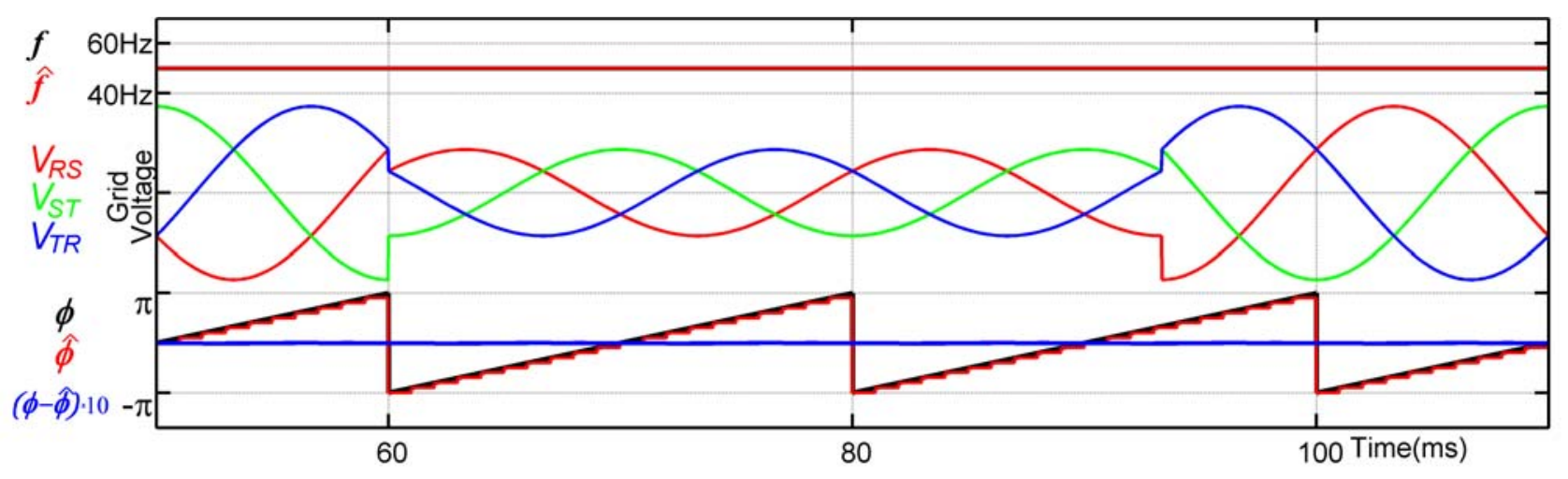

Fig. 4. Synchronizer performance without filter in a voltage sag (50\%) without harmonics.

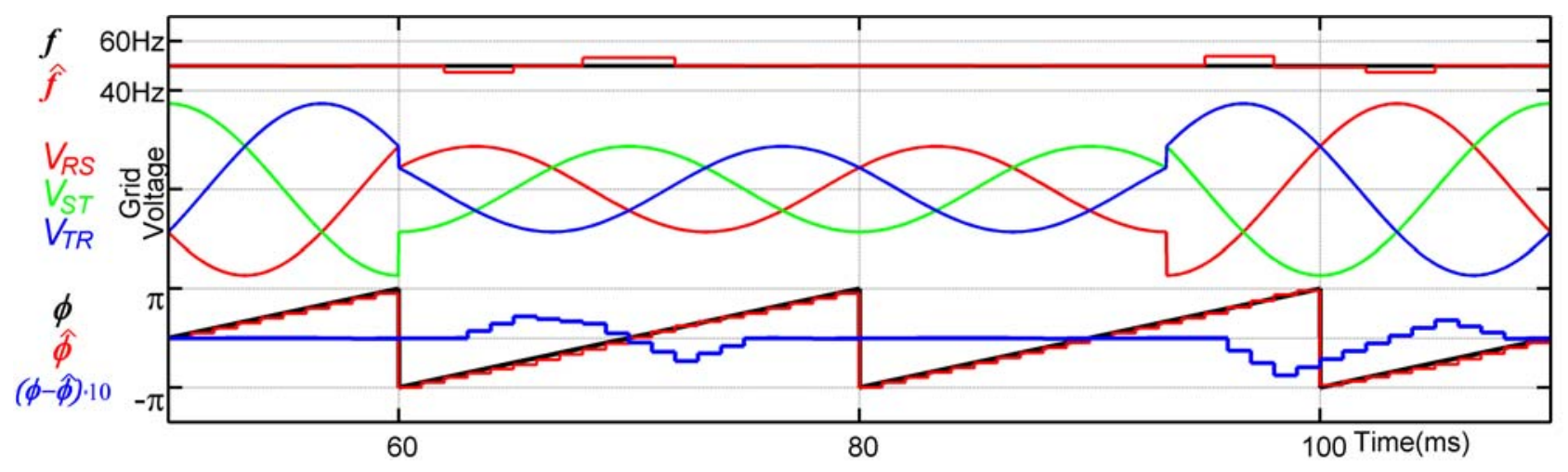

Fig. 5. Synchronizer performance with a filter in a voltage sag (50\%) without harmonics.

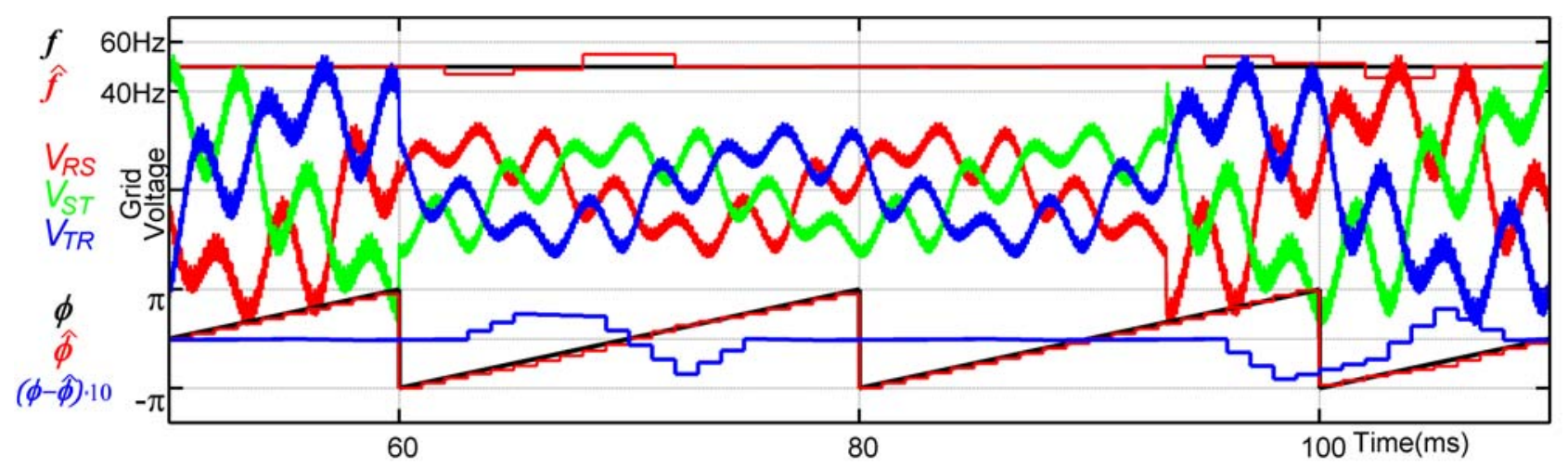

Fig. 6. Synchronizer performance under a voltage sag $(50 \%)$ in a grid polluted with harmonics $\left(25 \% 5^{\text {th }}\right.$ harm, $50 \% 7^{\text {th }}$ harm) and high frequency noise.

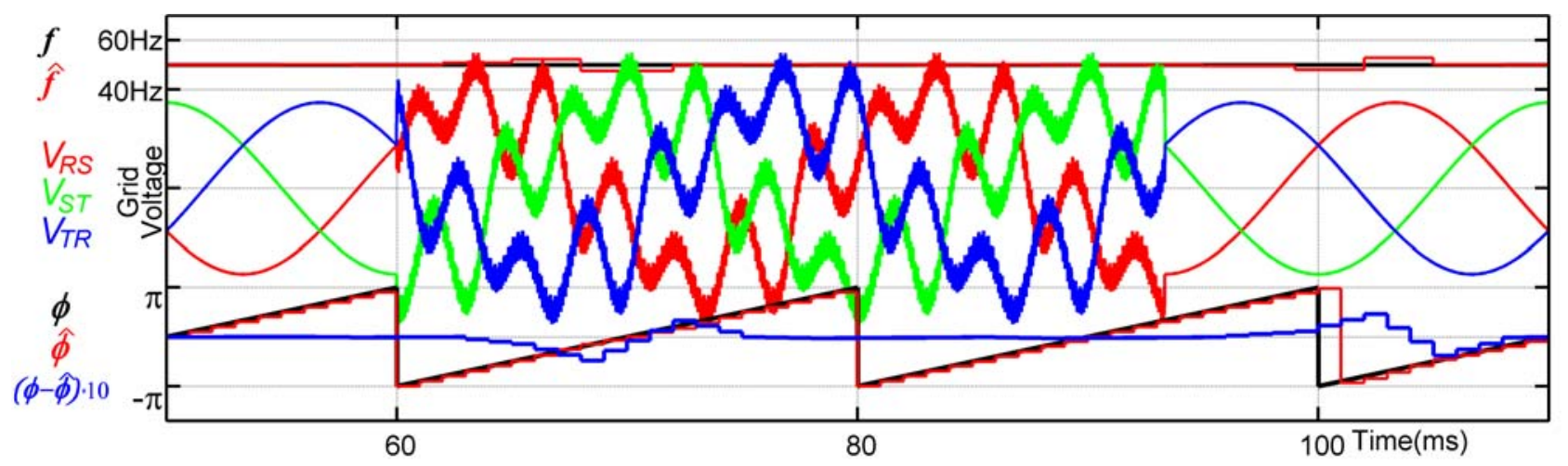

Fig. 7. Synchronizer performance when the grid gets polluted with harmonics $\left(25 \% 5^{\text {th }}\right.$ harm, $50 \% 7^{\text {th }}$ harm $)$ and high frequency noise. 


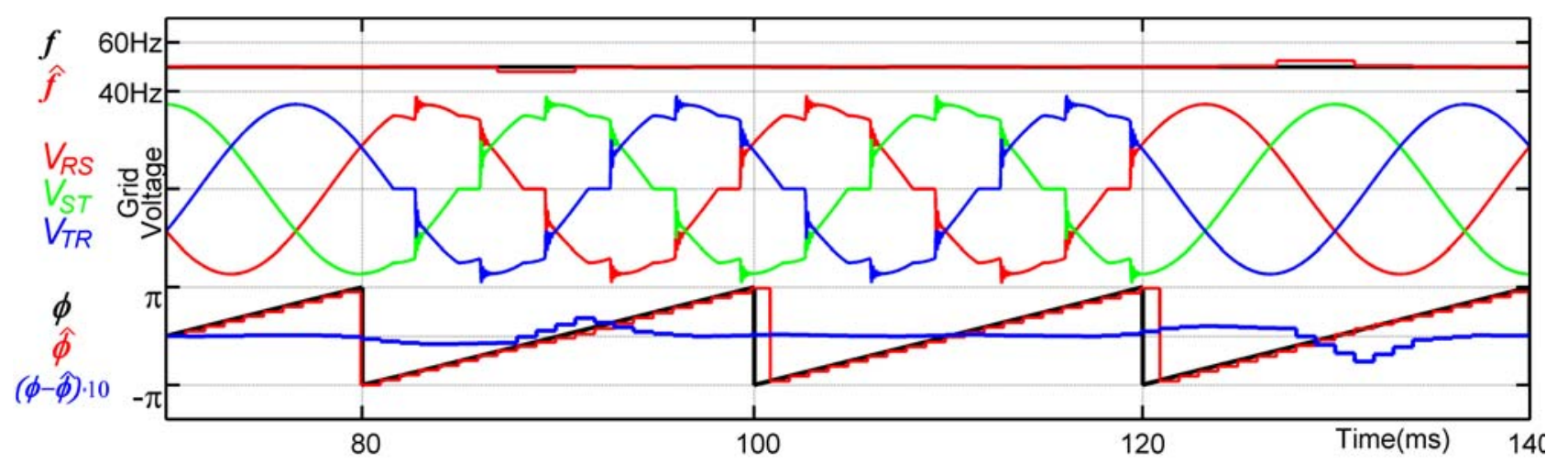

Fig. 8. Synchronizer performance when the grid gets polluted with harmonics due to a high power rectifier.

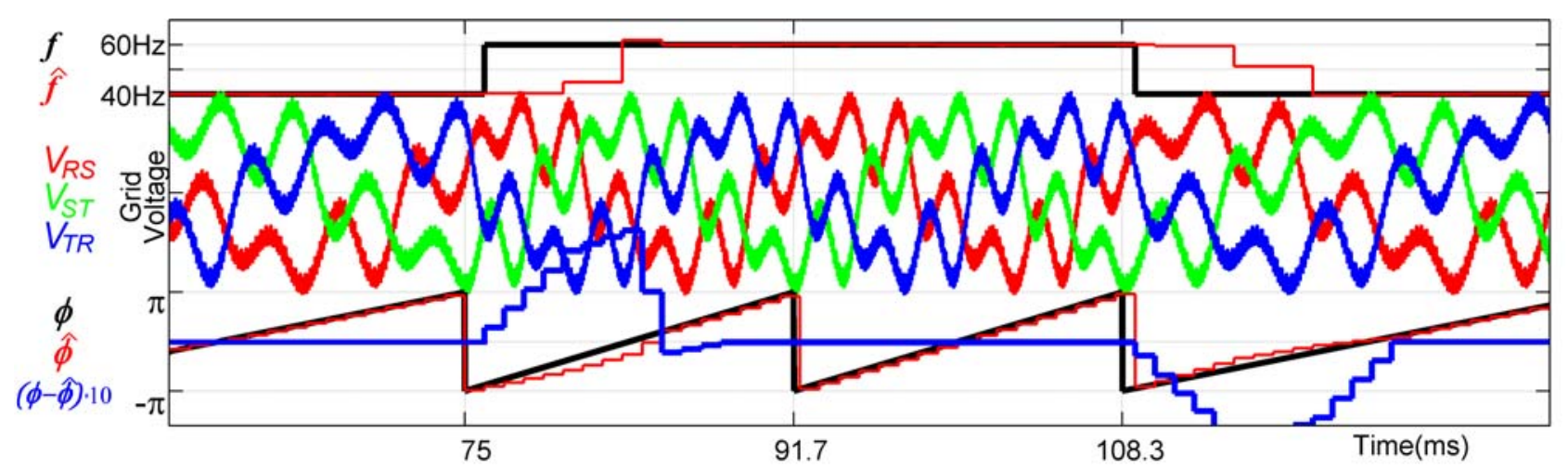

Fig. 9. Synchronizer performance with a grid polluted with harmonics $\left(25 \% 5^{\text {th }}\right.$ harm, $50 \% 7^{\text {th }}$ harm $)$ and high frequency noise, when the grid is subjected to a step change.

The proposed synchronism method was tested with extreme disturbances on the grid voltage. In all conditions the estimated grid phase angle converges to the real value in less than one grid period, showing its robustness.

\section{Conclusions}

The paper discusses the main disturbances occurring within a weak grid in what concerns phase detection in order to get an accurate synchronism between an electronic converter and the grid. It is an important issue in the domain of electrical generation as the control of power flow demands for an accurate control of phase angle between the both systems.

The authors proposed a method to handle the phase disturbances and presented a synchronizer with results carried out within Saber Designer environment that validate the adopted approach.

\section{References}

[1] A. V. Oppenheim, R. W. Schafer, and J. R. Buck, Discrete-Time Signal Processing, $2^{\text {nd }}$ edition; PrenticeHall, (1999).

[2] S. Sakamoto, T. Izumi, T. Yokoyama, and T. Haneyoshi, "A new method for digital PLL control using estimated quadrature two phase frequency detection", in Proc. of the Power Conversion Conference, Osaka, 2002, Vol. 2, pp. 671 -676.

[3] R. Weidenbrug, F. P. Dawson, and R. Bonert, "New synchronization method for thyristor power converters connected to weak AC systems", IEEE Trans. on Industrial Electronics, Vol. 40, No 5, pp. 505-511, Oct. 1993.

[4] J. Svensson, "Synchronization methods for gridconnected voltage source converters", IEE Proceedings Generation, Transmission and Distribution, Vol. 148, $\mathrm{N}^{\mathrm{o}} 3$, pp. 229-235, May 2001.

[5] O. Vainio, and S. J. Ovaska, "Noise reduction in zero crossing detection by predictive digital filtering", IEEE Transactions on Industrial Electronics, Vol. 42, $\mathrm{N}^{\mathrm{o}} 1$, pp. 58-62, Feb 1995. 